\title{
Curcumin suppresses LGR5(+) colorectal cancer stem cells by inducing autophagy and via repressing TFAP2A-mediated ECM pathway
}

\author{
Xiaohong Mao $^{1} \cdot$ Xin Zhang $^{2} \cdot$ Xiaowei Zheng $^{3} \cdot$ Yongwu Chen $^{4} \cdot$ Zixue Xuan $^{1} \cdot$ Ping Huang $^{1}$
}

Received: 20 November 2020 / Accepted: 3 March 2021 / Published online: 13 March 2021

(c) The Author(s) 2021

\begin{abstract}
Colorectal cancer stem cells (CSCs) have the potential for self-renewal, proliferation, and differentiation. And LGR5 is a stem cell marker gene of colorectal cancer. Curcumin can suppress oncogenicity of many cancer cells, yet the effect and mechanism of curcumin in LGR5(+) colorectal cancer stem cells (CSCs) have not been studied. In this study, we studied the effect of curcumin on LGR5(+) colorectal CSCs using the experiments of tumorsphere formation, cell viability and cell apoptosis. Then autophagy analysis, RNA-Seq, and real-time PCR were used to identify the mechanism responsible for the inhibition of LGR5(+) colorectal CSCs. Our results showed that curcumin inhibited tumorsphere formation, decreased cell viability in a dose-dependent manner, and also promoted apoptosis of LGR5(+) colorectal CSCs. Next, we found curcumin induced autophagy of LGR5(+) colorectal CSCs. When LGR5(+) colorectal CSCs were co-treated with curcumin and the autophagy inhibitor (hydroxychloroquine), curcumin-induced cell proliferation inhibition decreased. In addition, we also found that curcumin inhibited the extracellular matrix (ECM)-receptor interaction pathway via the downregulation of the following genes: GP1BB, COL9A3, COMP, AGRN, ITGB4, LAMA5, COL2A1, ITGB6, ITGA1, and TNC. Further, these genes were transcriptionally regulated by TFAP2A, and the high expression of TFAP2A was associated with poor prognosis in colorectal cancer. In conclusion, curcumin suppressed LGR5(+) colorectal CSCs, potentially by inducing autophagy and repressing the oncogenic TFAP2A-mediated ECM pathway.
\end{abstract}

\section{Graphic abstract}

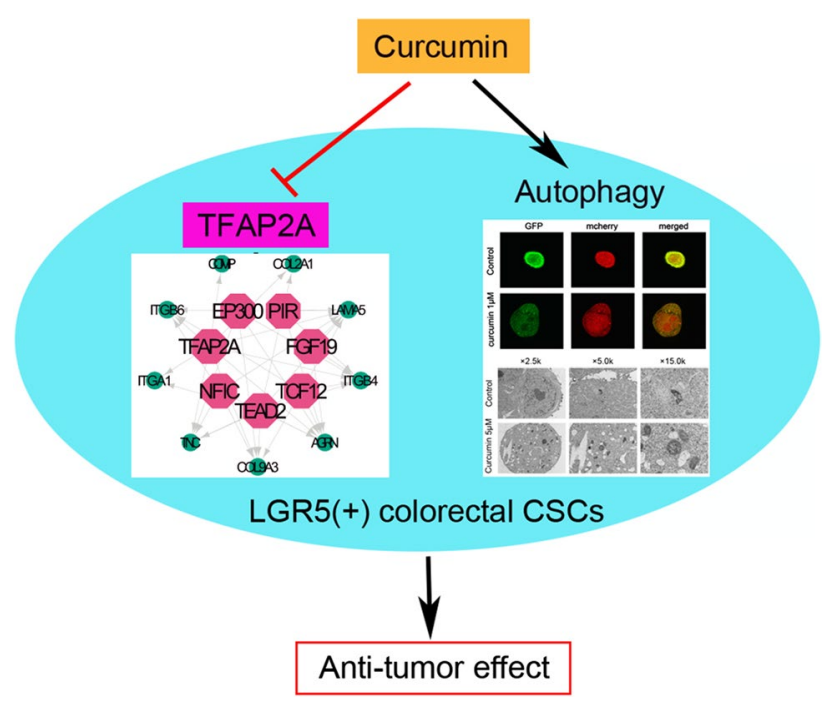

Xiaohong Mao and Xin Zhang have contributed equally to this work.

Extended author information available on the last page of the article 
Keywords Colorectal cancer · Curcumin · Cancer stem cells · Autophagy · LGR5 · TFAP2A

\section{Introduction}

Colorectal cancer (CRC) is the world's fourth most deadly cancer, and the second and third most common cancer diagnosed in women and men, respectively [1]. Advanced stage or metastatic CRC have limited sensitivity to conventional antitumor agents, because a subpopulation of colorectal cancer stem cells (CSCs) displays self-renewal, chemoresistance, and metastatic potential [2-4]. At present, more studies have confirmed that CSCs adversely affect the outcomes of CRC patients, yet agents targeting CSCs are not currently available in clinic [5].

Curcumin is a polyphenol extracting from Curcuma longa. It has been demonstrated that curcumin possesses extensive therapeutic activities against multiple ailments, such as inflammation, metabolic syndrome, liver disease, arthritis, and neurodegenerative disease [6]. In the recent years, researchers found that curcumin has played an important role in cancer prevention and treatment [7-9]. For instance, curcumin induced apoptosis of castration-resistant prostate cancer cells, partially dependent on its iron-chelating properties [10]; curcumin could overcome gefitinibresistance in nonsmall-cell lung cancer cells via inducing autophagy-related cell death [11]. Other studies indicated curcumin suppressed oncogenicity of human colon cancer cells, through covalent modification of SIRT1 at the cysteine 67 residue and the proteasomal degradation of oncogenic SIRT1 [12]. However, there have been no studies elucidating the effect of curcumin and its related mechanisms in colorectal CSCs.

In addition, recent studies have revealed that LGR5 is one of the few cell surface markers used to identify and isolate actively cycling stem cells in the colonic crypt [13]. Furthermore, it is widely known that colorectal CSCs are best studied in vitro using human LGR5(+) colorectal cancer cells [14]. For example, Morgan RG thought there is the plasticity or redundancy of LGR5 + cells in intestinal cancer progression and targeting LGR5 may be a great therapeutical strategy for CRC.

To investigate the effects and the mechanism of curcumin on LGR5(+) colorectal CSCs, we used vitro studies including the tumorsphere formation, cell viability and cell apoptosis, to illuminate the effect of curcumin on LGR5(+) colorectal CSCs. Then, we used autophagy analysis, RNASeq, and real-time PCR to identify the mechanism of the inhibition in LGR5(+) colorectal CSCs.

\section{Materials and methods}

\section{Cell line and reagents}

SW620 cell line (ATCC CCL-227) was obtained from the American Type Culture Collection (ATCC, Manassas, VA, USA). Recombinant human epidermal growth factor (EGF, Lot No.AF-100-15B) and recombinant human basic fibroblast growth factor (FGF, Lot No.AF-100-18B) were purchased from PeproTech, Inc. (Rocky Hill, NJ, USA). Dulbecco's modified Eagle's medium (DMEM)/F12 medium, B-27 (Lot No.17504-044), and N-2 (Lot No.17502-048) supplements and recombinant human leukemia inhibitory factor (LIF, Lot No. PHC9484) were purchased from Gibco (Life Technologies, Corp., Carlsbad, CA, USA). Curcumin (Lot No.C1386), hydroxychloroquine (HCQ, Lot No. PHR1782) and rapamycin (Lot No. V900930) were purchased from Sigma (Sigma-Aldrich, USA).

\section{Colorectal CSCs}

SW620 cells $\left(5 \times 10^{3} /\right.$ well $)$ were seeded in flat-bottom ultra-low attachment culture plates (Corning, NY, USA) in DMEM/F12 medium supplemented with $20 \mathrm{ng} / \mathrm{mL}$ EGF, $20 \mathrm{ng} / \mathrm{mL}$ FGF, B-27 $(1 \times)$ supplement, N-2 $(1 \times)$ supplement, and $10 \mathrm{ng} / \mathrm{mL}$ LIF. Then, the cells were incubated at $37{ }^{\circ} \mathrm{C}$ in a humidified incubator with $5 \% \mathrm{CO}_{2}$ and $95 \%$ air [15]. After seven to ten days, sphere formations of colorectal CSCs were observed under DMi8 inverted light microscope (Leica Microsystems, Wetzlar, Germany).

\section{Fluorescence-activated cell sorting}

Cells were labeled with anti-LGR5 MicroBeads (Lot No.5191031303, Miltenyi Biotec, Bergisch Gladbach, Germany) and then LGR5(+) cells were enriched by magnetic activated cell sorting. Fluorescence-activated cell sorting was performed using BD FACSAria Cell Sorter (BD Biosciences, San Jose, CA, USA) [16].

\section{Western blotting}

The total protein of LGR5(+) SW620 CSCs, LGR5(-) SW620 CSCs, and SW620 CSCs was extracted, and $50 \mu \mathrm{g}$ of the total protein was used for electrophoresis. We transferred the electrophoresis products onto polyvinylidene fluoride membranes, and the membranes were blocked with 5\% skim milk for one hour. After washing 
with Tris-buffered saline with Tween-20 (TBST, $1 \times$ ), the membrane was incubated with primary antibodies LGR5 (1:5000, Lot No. Ab75850; Abcam, Cambridge, UK) and $\beta$-tubulin (1:500, Lot No.K200059M; Solarbio, China), and shaken at $4{ }^{\circ} \mathrm{C}$ overnight. Next, the polyvinylidene fluoride membranes were washed thrice with TBST, incubated with a secondary antibody at room temperature for $1 \mathrm{~h}$ and washed with TBST again. Finally, immunoreactive products were visualized using enhanced chemiluminescence (Tanon-4200, China) and quantified using ImageJ software (version 1.50, National Institutes of Health).

\section{Sphere formation, cell viability, and cell apoptosis}

LGR5(+) colorectal CSCs were incubated with different concentrations of curcumin $(1 \mu \mathrm{M}, 5 \mu \mathrm{M}, 25 \mu \mathrm{M})$ for $48 \mathrm{~h}$. Then, sphere formations of different groups were observed under inverted light microscope. The culture medium (DMEM/F12) was then discarded and $100 \mu \mathrm{L}$ fresh culture medium was added, along with $10 \mu \mathrm{L}$ CCK- 8 reagent (Cell Counting Kit-8, Lot No.CK04; Dojindo Molecular Technologies, Inc., Tokyo, Japan) into each well, and incubated at $37{ }^{\circ} \mathrm{C}$ for one hour. The optical density was measured at $450 \mathrm{~nm}$ using a microplate reader (Multiskan Spectrum, Thermo, USA).

In this study, the Annexin V-APC (PI) Apoptosis Analysis Kit (Lot No.AO2001-11P-H, Tianjin Sungene Biotech Co., Ltd., Tianjin, China) was used to analyze cell apoptosis[17]. After treated with curcumin $(5 \mu \mathrm{M})$ for $24 \mathrm{~h}$, the LGR5(+) colorectal CSCs were suspended in $100 \mu \mathrm{L}$ binding buffer, and stained with $5 \mu \mathrm{L}$ APC-conjugated Annexin V and $5 \mu \mathrm{L}$ propidium iodide for $15 \mathrm{~min}$ at room temperature in the dark. Finally, $400 \mu \mathrm{L}$ binding buffer was added, and apoptotic cells were analyzed by flow cytometry (BD Biosciences, San Jose, CA, USA).

\section{Autophagy analysis}

Because the green fluorescent protein (GFP) signals can be quenched and the monomeric red fluorescent protein (mRFP) signal is more stable in the acidic environment of the lysosome, autolysosomes, and autophagosomes were labeled with mRFP (red) or GFP (green), respectively[18]. The LGR5(+) colorectal CSCs were transfected with AdmCherry-GFP-LC3B $\left(2.51 \times 10^{10} \mathrm{PFU} / \mathrm{mL}\right)$, and treated with different concentrations of curcumin $(1 \mu \mathrm{M}$ and $5 \mu \mathrm{M})$ or rapamycin $(100 \mathrm{nM})$ for $48 \mathrm{~h}$. In this study, autophagy inducer rapamycin served as a positive control group. Then, the expression of mCherry and GFP was visualized using confocal fluorescence microscopy (Leica TCS SP8; Leica Microsystems) at $100 \times$ magnification. Autophagy flux was evaluated by observing the number of yellow and red puncta.
After treated with curcumin $(1 \mu \mathrm{M}$ and $5 \mu \mathrm{M})$ or rapamycin $(100 \mathrm{nM})$ for $24 \mathrm{~h}$, the LGR5(+) colorectal CSCs were washed twice with ice-cold PBS, fixed using $2.5 \%$ glutaraldehyde for $60 \mathrm{~min}$ at room temperature, and post-fixed in $1 \%$ osmium tetroxide for $30 \mathrm{~min}$. As described previously [19, 20], all samples were embedded in EPON resin and stained with uranyl acetate and lead citrate. The cells were observed under a transmission electron microscope (H-7650; Hitachi High-Technologies Corp., Tokyo, Japan).

According to our previous studies[18, 20], HCQ could inhibit tumor autophagy. To confirm the effect of curcumininduced autophagy on curcumin-mediated cell inhibition of LGR5(+) colorectal CSCs, cells were co-treated and incubated with curcumin $(5 \mu \mathrm{M})$ and HCQ $(10 \mu \mathrm{M})$; sphere formation and cell viability were then analyzed.

\section{RNA-Seq for gene analysis}

Total RNA of the LGR5(+) colorectal CSCs treated with and without curcumin $(5 \mu \mathrm{M})$, were extracted using TRIzol® Reagent (Invitrogen, Carlsbad, CA, USA) according to the manufacturer's instructions. To explore the mechanism of curcumin in LGR5(+) colorectal CSCs, RNA-Seq was performed. Firstly, Agilent 2100 Bioanalyzer (Agilent Technologies, Santa Clara, CA, USA) was used to confirm RNA quality, then RNA-Seq libraries were prepared from three biological replicates and sequenced on an Illumina HiSeq (Illumina, Inc., San Diego, CA, USA). Fastqc and RSEM were used to assess the quality of the reads and calculate the expression of the genes, respectively [21]. Subsequently, the edgeR software (version 3.30.3) was used and the differential expression was calculated by Gene Read Count [22]. The screening criteria for significantly differentially expressed genes were $\mathrm{FDR}<0.05$ and $\mid \log _{2} \mathrm{FCl} \geq 1$. These genes were analyzed by clustering expression patterns, and were classified according to the involved biological processes and molecular functions and associated cell components, using the Gene Ontology database. In addition, KOBAS (http:// kobas.cbi.pku.edu.cn/home.do) was used to analyze the pathway enrichment analysis using Kyoto Encyclopedia of Genes and Genomes (KEGG).

Next, Cytoscape plugin iRegulon was used to identify master regulators that targeted these significantly expressed genes [23]. Transcription factors (TFs) of the master regulators were identified when they overlapped with the significantly expressed gene signatures. As previously described, the algorithm was based on a typical ranking-and-recovery strategy [24], and all the default parameters were left unchanged while predicting TFs. In this experiment, TFs with normalized enrichment score and normalized enrichment score (NES) $\geq 6$ were set as the parameters to build the regulatory network. 


\section{TFAP2A mRNA expression and prognosis correlation in CRC}

A CRC cDNA microarray (MecDNA-HColA095Su01) containing 15 paired cancer and non-CRC tissues and 65 CRC tissues was purchased from Shanghai Outdo Biotech Company (Shanghai, China). The mRNA expression of TFAP2A and GAPDH was quantified with SYBR mixture (Takara Biotechnology, Co., Ltd., Dalian, China), using a real-time PCR machine (LightCycler 480 Instrument II, Roche Molecular Systems, Inc., Pleasanton, CA, USA). The primers used were the following: for TFPA2A, TFAP2A F: 5'-CGTGTCCCTGTCCAAGTCCAA-3' and TFAP2A R: 5'-GACCCGGAACTGAACAGAAGA-3' [25]; for human $\beta$-actin, Human $\beta$-actin-F1: 5'-GAA GAGCTACGAGCTGCCTGA- ${ }^{\prime}$ ' and human $\beta$-actin-R1: 5'- CAGACAGCACTGTGTTGGCG-3'.

To investigate the correlation between TFAP2A expression with overall survival in CRC, the expression of TFAP2A was divided into two categories: high expression $\left({ }_{\Delta} \mathrm{CT}<11.1\right)$ and low expression $\left({ }_{\Delta} \mathrm{CT} \geq 11.1\right)$, and the correlation was analyzed by the Kaplan-Meier method with a log-rank test or Wilcoxon test.

\section{Statistical analysis}

All data were expressed as the mean \pm standard deviation of at least three individual experiments. The data were analyzed by one-way analysis of variance (ANOVA) using the SPSS Statistics 26.0 (IBM, Armonk, NY, USA). The following values were considered statistically significant: ${ }^{*} p<0.05$, $* * p<0.01, * * * p<0.001$.

\section{Results}

\section{LGR5(+) colorectal CSCs were obtained}

Human LGR5(+) colorectal CSC models provide an opportunity to study the effect and mechanism of antitumor drugs. SW620 cells were cultured in flat-bottom ultra-low attachment culture plates in DMEM/F12 medium supplemented with $20 \mathrm{ng} / \mathrm{mL}$ EGF, $20 \mathrm{ng} / \mathrm{mL}$ FGF, B-27 (1×) supplement, $\mathrm{N}-2(1 \times)$ supplement, and $10 \mathrm{ng} / \mathrm{mL}$ LIF. After sphere formation was observed, cells were labeled with anti-LGR5 MicroBeads, then LGR5(+) cells were enriched by magnetic activated cell sorting. The results of western blotting revealed that LGR5 was highly expressed in LGR5(+) SW620 CSCs, but was hardly detected in LGR5(-) SW620 CSCs (Fig. 1a, b). In addition, the tumorsphere was better
Fig. 1 Identification of LGR5(+) colorectal CSCs. a The LGR5 protein expression of LGR5(+) SW620 CSCs, LGR5(-) SW620 CSCs, and SW620 CSCs was demonstrated by western blotting. b Relative expression levels of LGR5 in LGR5(+) SW620 CSCs, LGR5(-) SW620 CSCs, and SW620 CSCs. c Tumorsphere formation of LGR5(+) SW620 CSCs and LGR5(-) SW620 CSCs. $* * * p<0.001$
A
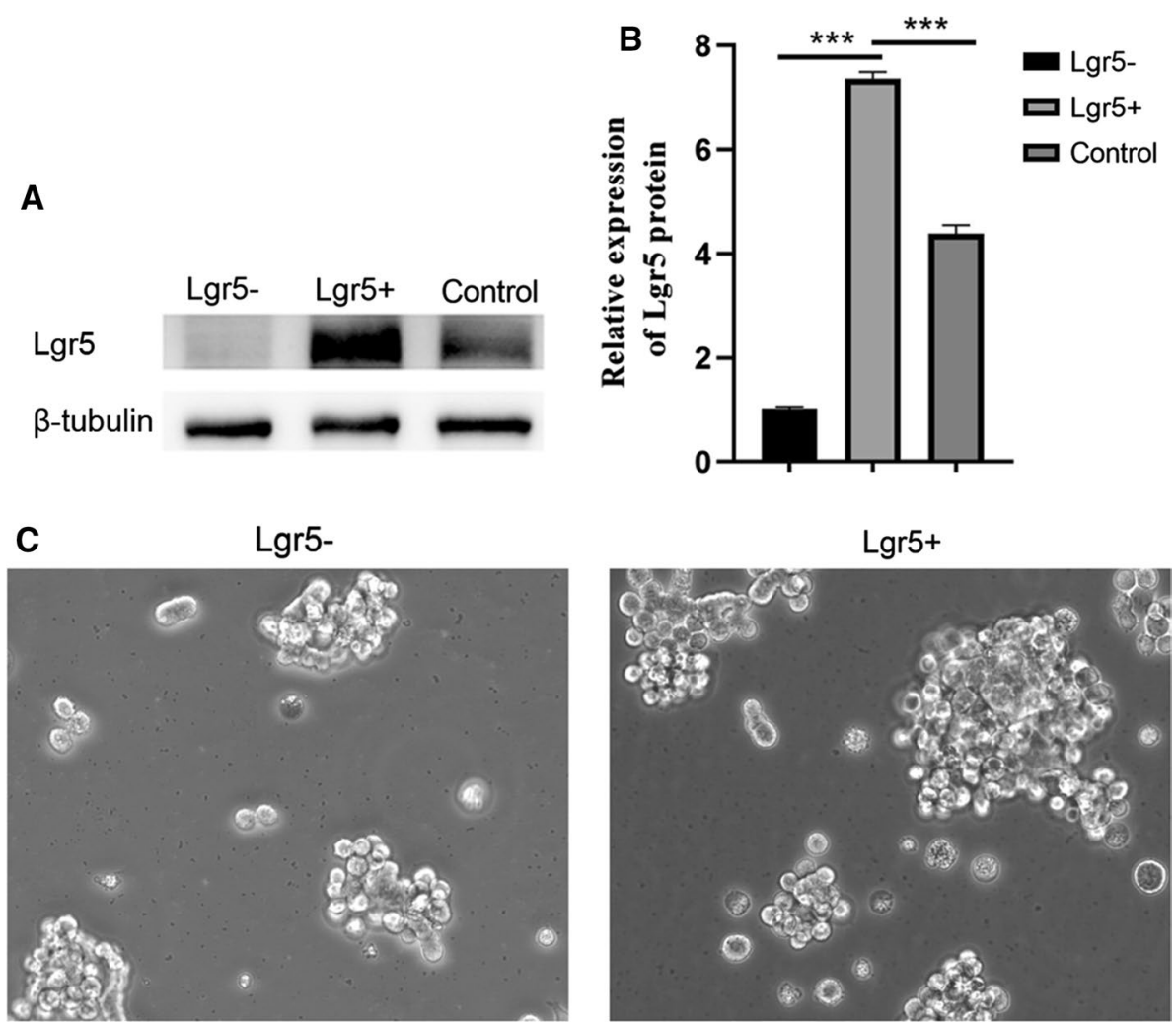
formed in LGR5(+) SW620 CSCs than in LGR5(-) SW620 CSCs (Fig. 1c).

\section{Curcumin inhibited tumorsphere formation, decreased cell viability and promoted apoptosis of LGR5(+) colorectal CSCs}

To confirm the effect of curcumin in LGR5(+) colorectal CSCs, LGR5(+) SW620 CSCs were treated with curcumin $(1 \mu \mathrm{M}, 5 \mu \mathrm{M}$, and $25 \mu \mathrm{M})$. We found that curcumin reduced tumorsphere formation in a dose-dependent manner (Fig. 2a). The results of cell viability analysis showed that curcumin also inhibited the growth of LGR5(+) colorectal $\mathrm{CSCs}$ in a dose-dependent manner (Fig. 2b).
In apoptosis analysis, the apoptosis rate of cells treated with curcumin $(5 \mu \mathrm{M})$ was $39.36 \pm 2.36 \%$, whereas the apoptosis rate of control cells was $5.07 \pm 0.62 \%$ (Fig. $2 \mathrm{c}, \mathrm{d}$ ).

\section{Curcumin induced autophagy of LGR5(+) colorectal CSCs}

Although it is known that curcumin can induce autophagy in many tumor cells, whether curcumin can induce autophagy of LGR5(+) colorectal CSCs was unclear. Thus, we transfected the curcumin $(1 \mu \mathrm{M}$ and $5 \mu \mathrm{M})$ treated-LGR5(+) colorectal CSCs with Ad-mRFP-GFP-LC3 adenovirus, and then detected the two different fluorescent signals to monitor the autophagic flux status post-treatment with curcumin. In this study, autophagy inducer rapamycin served as a positive
A

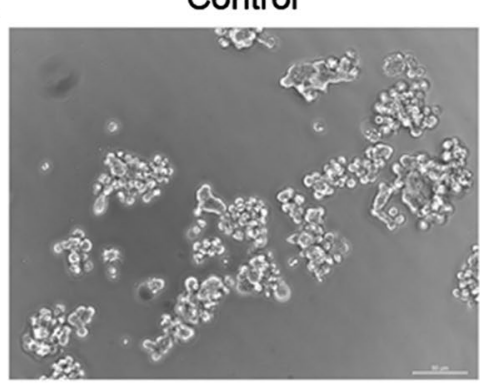

Curcumin $5 \mu \mathrm{M}$

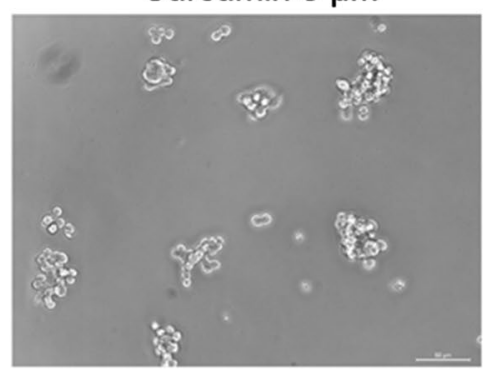

C

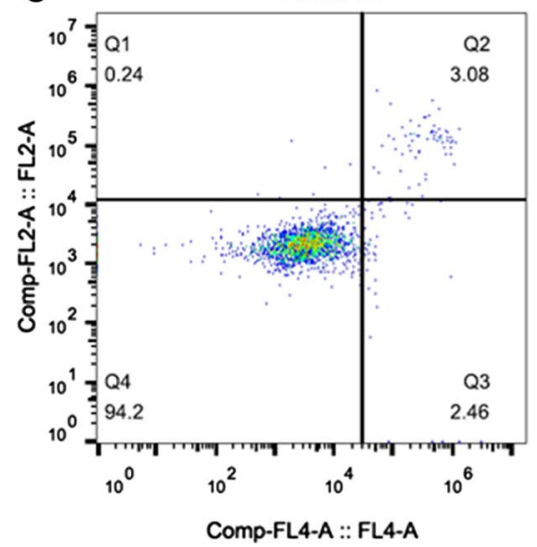

Curcumin $1 \mu \mathrm{M}$

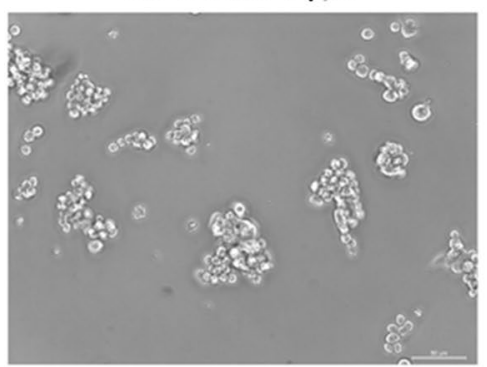

Curcumin $25 \mu \mathrm{M}$
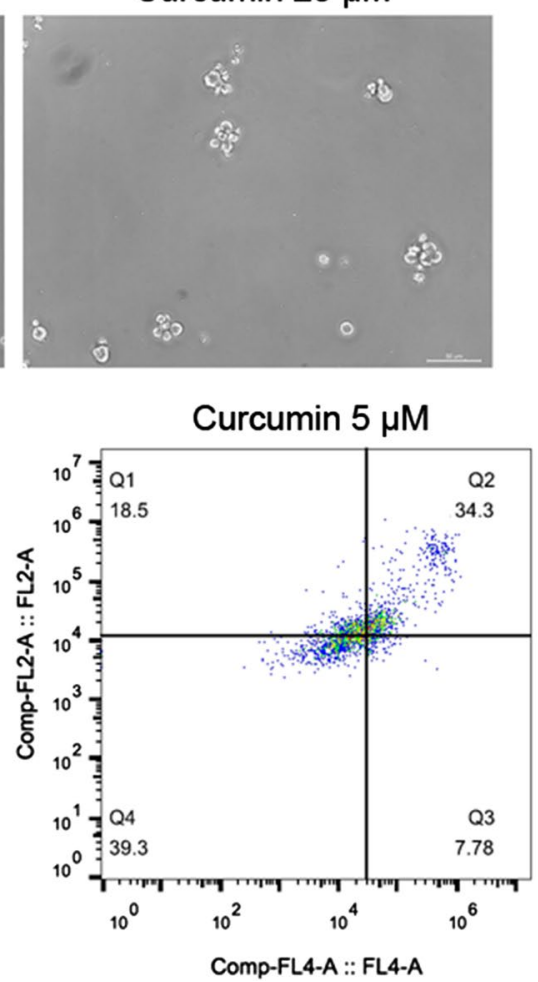

B

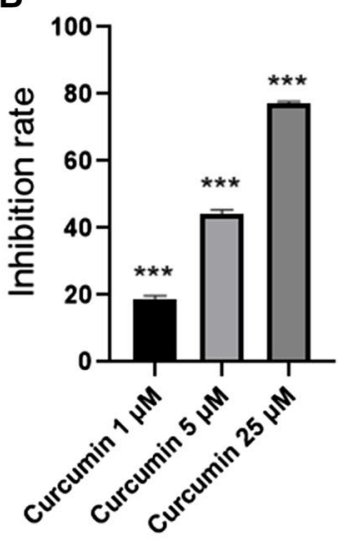

D

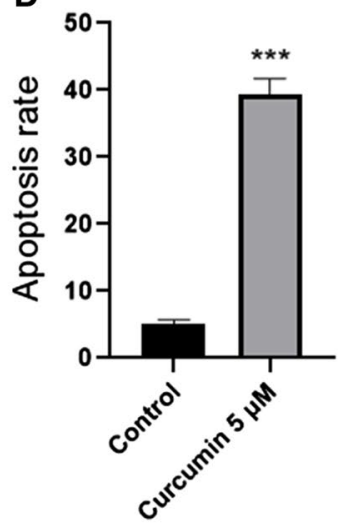

Fig. 2 Curcumin suppressed LGR5(+) colorectal CSCs. a Curcumin reduced tumorsphere formation of LGR5(+) colorectal CSCs in a dose-dependent manner. b Cell viability showed that curcumin inhib- ited cell growth of LGR5(+) colorectal CSCs in a dose-dependent manner. c, d Cell apoptosis analysis of LGR5(+) colorectal CSCs treated with curcumin $(5 \mu \mathrm{M}) . * * * p<0.001$ 
control group, then we found both yellow and red puncta were increased in cells treated with either curcumin or rapamycin, indicating that curcumin can induce autophagy in LGR5(+) colorectal CSCs (Fig. 3). In addition, we found that the degree of autophagy was proportional to the concentration of curcumin. These results were consistent with the data from transmission electron micrographs (Fig. 4), suggesting that curcumin induced autophagy.

As shown in Fig. 5a, co-treatment with curcumin $(5 \mu \mathrm{M})$ and HCQ $(10 \mu \mathrm{M})$ can increase the tumorsphere formation of LGR5(+) colorectal CSCs, compared to mono-treatment with curcumin. In the CCK-8 assay, inhibition rate decreased to $41.83 \%$ upon co-treatment with curcumin and HCQ, versus $66.12 \%$ upon mono-treatment with curcumin (Fig. 5b). Therefore, these results suggest that curcumin-mediated cell inhibition may be associated with curcumin-induced autophagy.

\section{Curcumin downregulated the oncogenic TFAP2A-mediated ECM pathway}

In order to identify mechanisms of curcumin in LGR5(+) colorectal CSCs, RNA-Seq was used, and the result revealed that there were 1098 significantly differentially expressed genes in curcumin-treated cells and control cells, with 363 upregulated and 735 downregulated
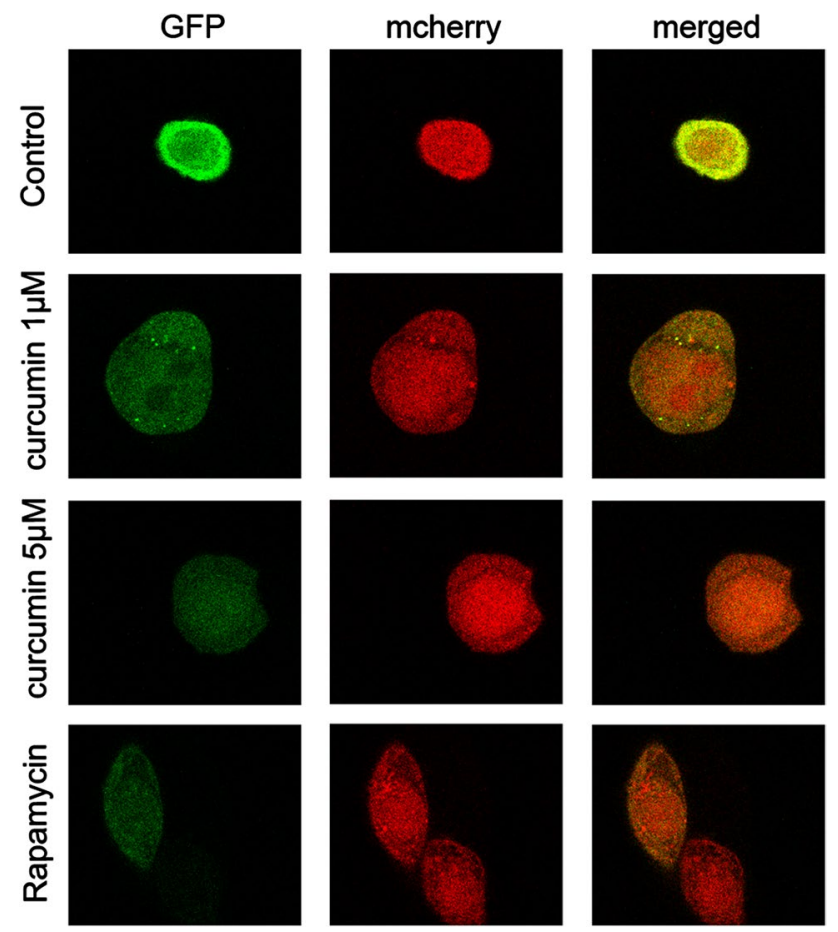

Fig. 3 After the cells were treated with different concentrations of curcumin $(1 \mu \mathrm{M}$ and $5 \mu \mathrm{M})$, or rapamycin $(100 \mathrm{nM})$, autophagy flux was evaluated by the mCherryEGFP-LC3 assay. Autolysosomes and autophagosomes were labeled with mRFP or GFP, respectively

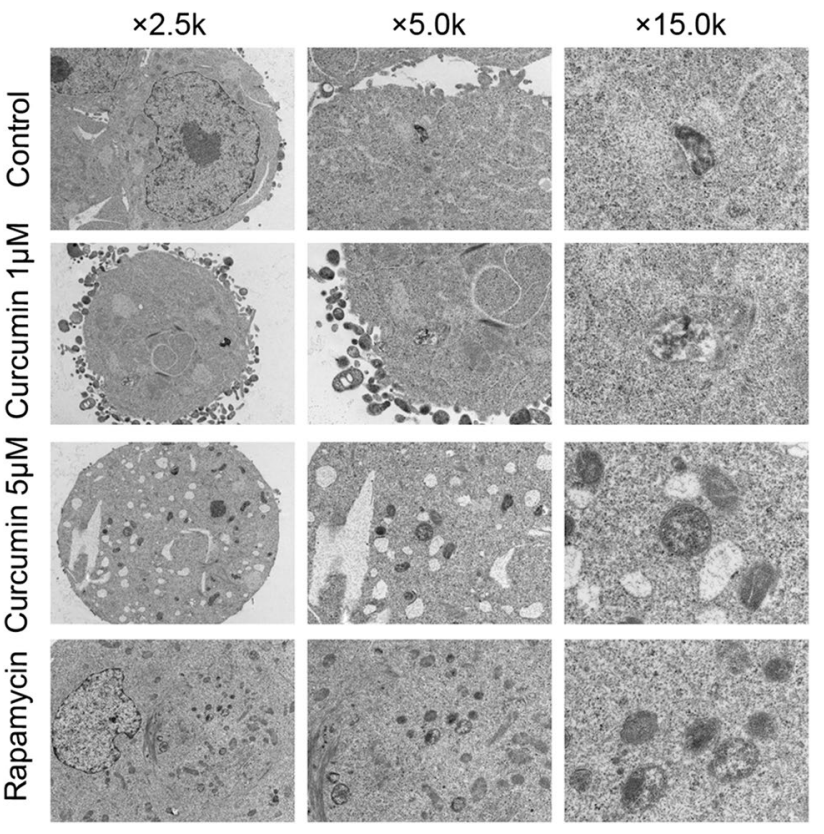

Fig. 4 Cells were observed under a transmission electron microscope after the cells were treated with different concentrations of curcumin $(1 \mu \mathrm{M}$ and $5 \mu \mathrm{M})$, or rapamycin $(100 \mathrm{nM})$

genes. These significantly differentially expressed genes between curcumin-treated cells and control cells were shown in Fig. 6a, and heat map of significantly differentially expressed genes in the top six pathways was shown in Fig. 6b.

KEGG pathway analyses revealed differential expression in curcumin-treated LGR5(+) cells involved in 'Pathways in cancer,' 'PI3K-Akt signaling pathway,' 'MAPK signaling pathway,' 'Wnt signaling pathway,' 'NF-kappa B signaling pathway,' 'ECM-receptor interaction,' and 'Lysosome' (Data not shown). In addition, there were ten significantly negatively expressed genes involved in ECM-receptor interaction, including GPIBB, COL9A3, COMP, AGRN, ITGB4, LAMA5, COL2A1, ITGB6, ITGA1, and $T N C$ (Fig. 6c, d). Using Kaplan-Meier Plotter (www. kmplot.com), we found that the ECM-receptor interaction pathway included these ten significantly negatively expressed genes and were associated with shorter survival of CRC patients (hazard ratios, HR $=2.15(0.99-4.69)$; logrank $p=0.049$ ) (Fig. 6E).

The results of RNA-Seq also revealed that the expression of LGR5 and TFAP2A in LGR5(+) colorectal CSCs treated with curcumin was significantly downregulated (Fig. 7a, b). Then, we used Cytoscape plugin iRegulon to identify the master regulators that targeted the aforementioned genes, and found the NES of TFAP2A was at 6.802, and as shown in the regulatory network, is a transcription factor. In addition, TFAP2A overlapped with nine out of ten genes, with the exception of GPIBB (Fig. 7c, d). 
Fig. 5 Effect of curcumininduced cell proliferation inhibition was decreased on co-treatment of cells with the autophagy inhibitor, hydroxychloroquine (HCQ). a Sphere formation assay of LGR5(+) colorectal CSCs mono-treated with curcumin $(5 \mu \mathrm{M})$ or cotreated with HCQ $(10 \mu \mathrm{M})$. b In the CCK- 8 assay, inhibition rate of LGR5(+) colorectal CSCs mono-treated with curcumin $(5 \mu \mathrm{M})$ or co-treated with HCQ $(10 \mu \mathrm{M}) . * * * p<0.001$
A

Control

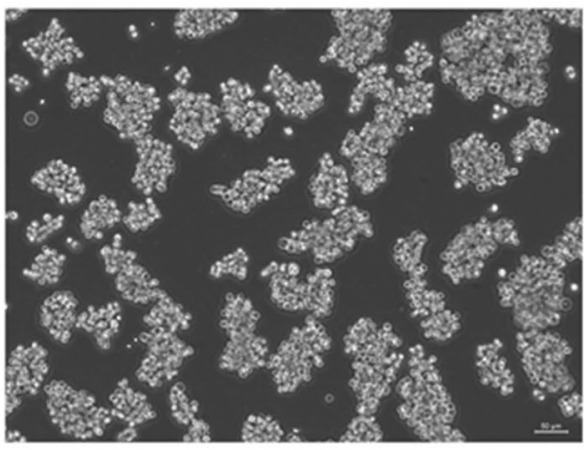

Curcumin $5 \mu \mathrm{M}+\mathrm{HCQ} 10 \mu \mathrm{M}$

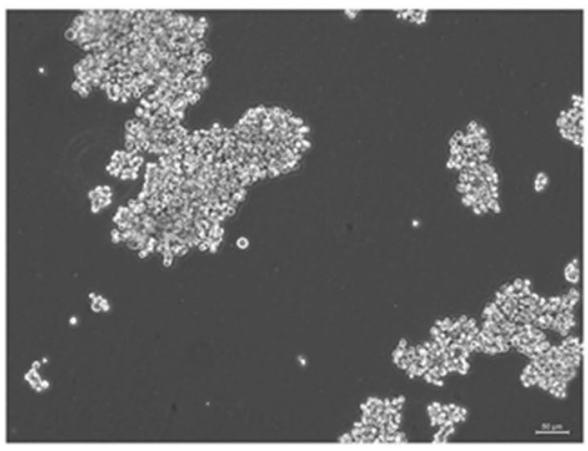

Curcumin $5 \mu \mathrm{M}$
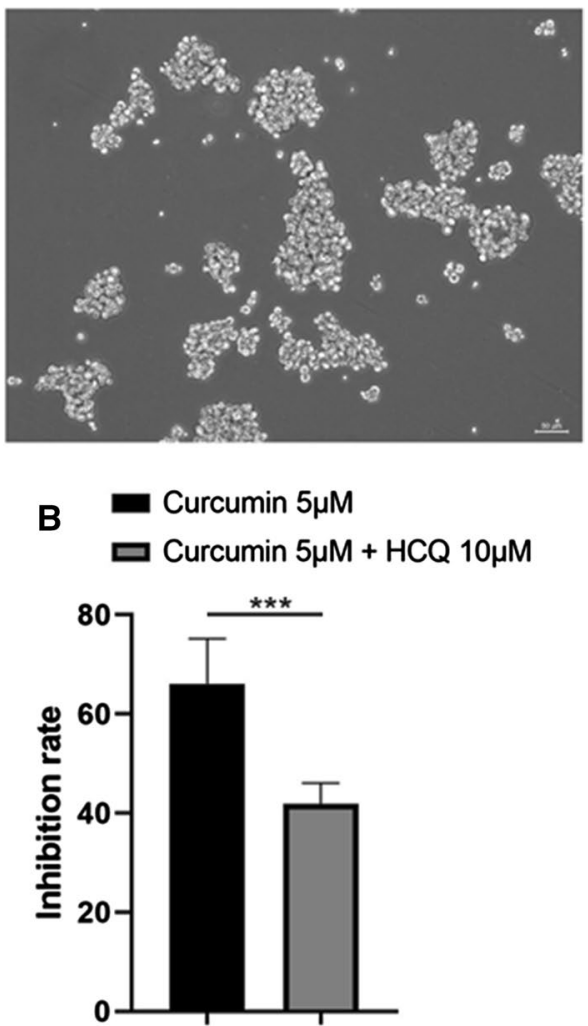

\section{TFAP2A was associated with poor survival in patients with CRC}

To investigate the role of TFAP $2 A$ in CRC, the mRNA expression of TFAP2A in CRC and noncancerous colorectal tissues was quantified using real-time PCR. Our results showed higher TFAP2A mRNA expression in CRC tissues compared with that in noncancerous colorectal tissues, TFAP2A mRNA expression was not associated with age, gender, grade, and TNM stage (Table 1). After the expression of TFAP $2 A$ was divided into high and low expression categories, we further assessed the correlation between TFAP2A mRNA expression and survival of patients, and found that higher expression of TFAP2A was correlated with poor overall survival of CRC patients (Fig. 7e, $p<0.05$ ).

\section{Discussion}

Colorectal cancer (CRC) is the world's fourth most deadly cancer, and evidence shows that rare CSCs have the potential for self-renewal, proliferation, and differentiation in CRC [26]. LGR 5 was originally isolated from colon cancer cells, where 56\% LGR5(+) cells were observed in colorectal CSCs [27]. Importantly, few LGR5(+) colorectal CSCs could grow a tumor with the same phenotype as that of original cells, and silencing the LGR5 gene could increase the apoptosis of tumor cells [13]. Therefore, the importance of LGR5 as a stem cell marker gene of CRC is already well established [28], and we used LGR5(+) colorectal CSCs to investigate the effect and mechanism of curcumin.

In this study, we observed that curcumin inhibited tumorsphere formation, decreased cell viability in a dosedependent manner. Curcumin also promoted apoptosis of LGR5(+) colorectal CSCs. In addition, curcumin can induce autophagy in many tumor cells. For example, curcumin inhibited proliferation, induced the autophagy and apoptosis in gastric cancer cells [29]. Similarly, our data suggest that curcumin increased tumor cell death partly by inducing autophagy, because the effect of curcumin-induced cell proliferation inhibition was decreased by co-treatment with the autophagy inhibitor, HCQ. Therefore, these results reveal that curcumin-induced autophagy may contribute to the antitumor effects of curcumin on LGR5(+) colorectal CSCs.

Next, the RNA-Seq data showed that curcumin decreased the expression of $G P 1 B B, C O L 9 A 3, C O M P, A G R N$, ITGB4, LAMA5, COL2A1, ITGB6, ITGA1, and TNC in the ECM-receptor interaction pathway, which is also associated with shorter survival of CRC patients. As previously reported, the ECM acts as a structural support and provides stem cells with signals to regulate its function via reciprocal interactions between cells and the components of the 


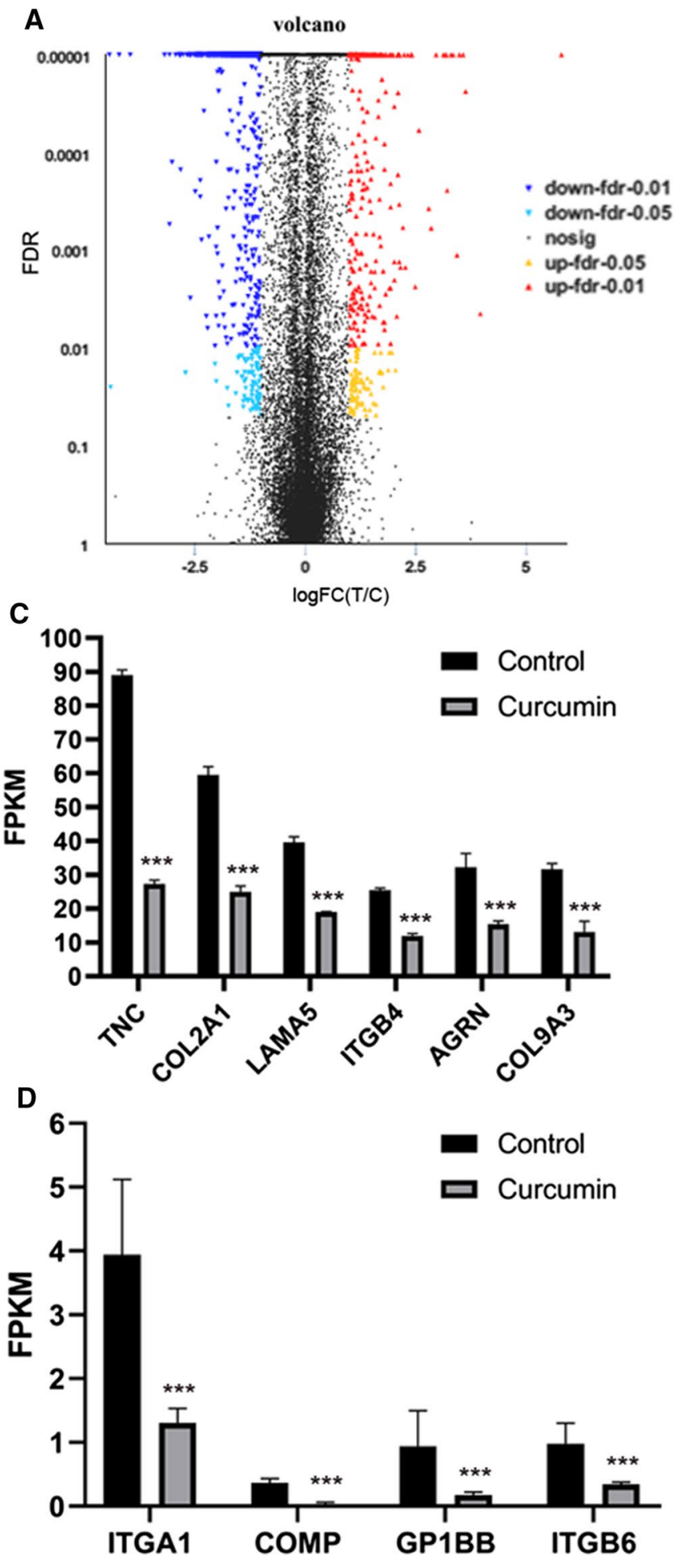

Fig. 6 RNA-Seq analysis. a The volcano plot of the significantly differentially expressed genes between curcumin-treated cells and control cells. b Heat map of significantly differentially expressed genes in the top six pathways. c, $\mathbf{d}$ Ten significantly negatively expressed genes involved in ECM-receptor interaction, including $G P 1 B B$,
B

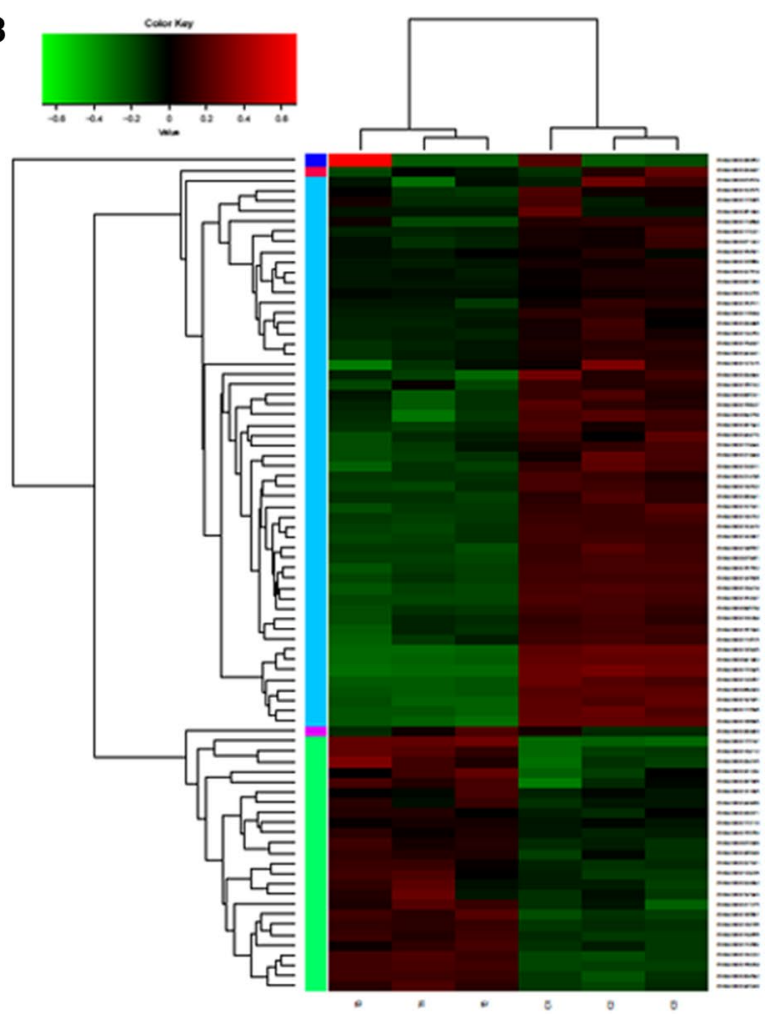

E 'ECM-receptor interaction' pathway

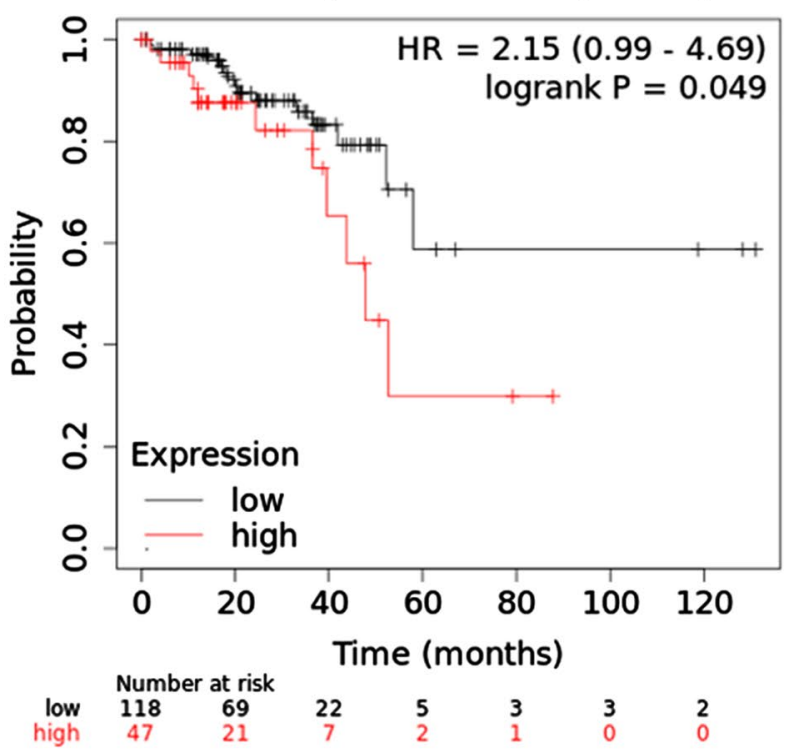

COL9A3, COMP, AGRN, ITGB4, LAMA5, COL2A1, ITGB6, ITGA1, and $T N C$. e Kaplan-Meier survival curves of ECM-receptor interaction pathway including the ten significantly negatively expressed genes in CRC patients. *p $p<0.05, * * p<0.01, * * * p<0.001$ 

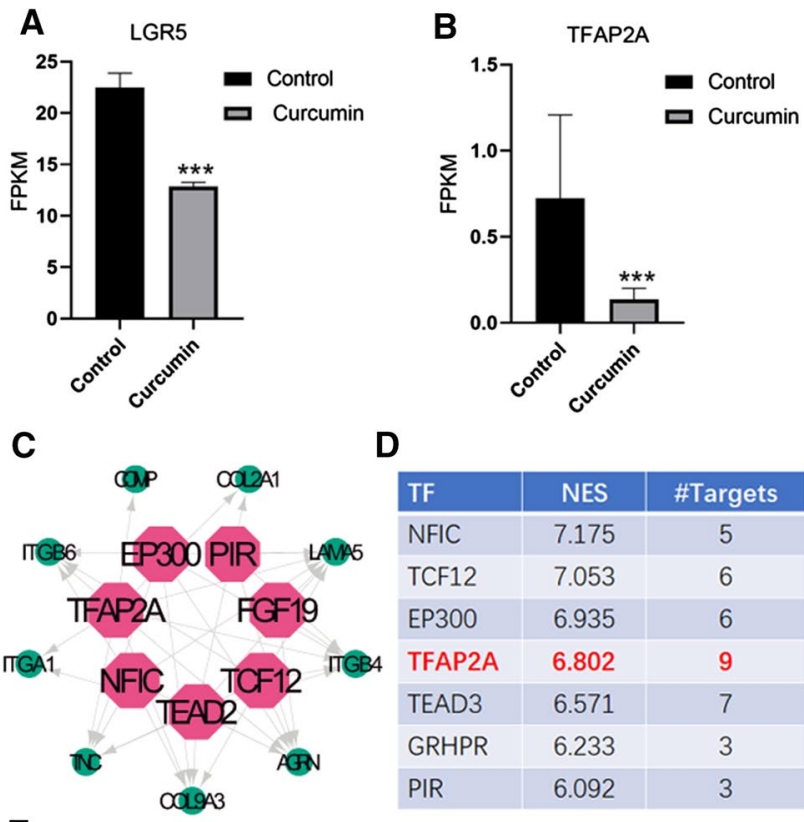

D

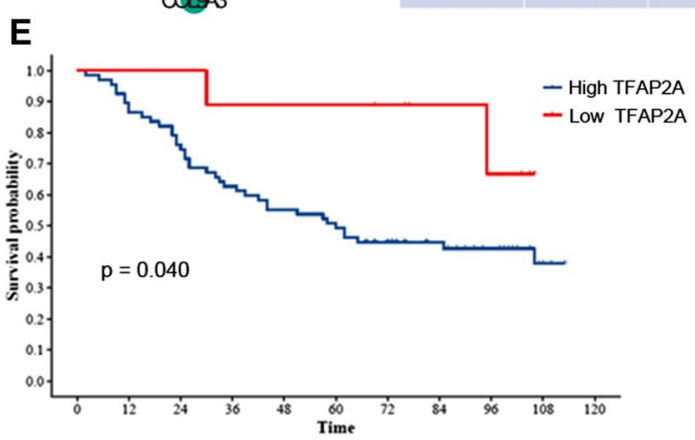

\begin{tabular}{|l|c|c|}
\hline TF & NES & \#Targets \\
\hline NFIC & 7.175 & 5 \\
\hline TCF12 & 7.053 & 6 \\
\hline EP300 & 6.935 & 6 \\
\hline TFAP2A & 6.802 & 9 \\
\hline TEAD3 & 6.571 & 7 \\
\hline GRHPR & 6.233 & 3 \\
\hline PIR & 6.092 & 3 \\
\hline
\end{tabular}

Fig. 7 Curcumin suppressed LGR5(+) colorectal CSCs by transcriptionally repressing oncogenic TFAP $2 A$. a Curcumin decreased the expression of LGR5. b Curcumin decreased the expression of $T F A P 2 A$. c Cytoscape plugin iRegulon was used to identify master regulators of targeted genes, and TFAP $2 A$ overlaps with nine genes in the regulatory network. d The normalized enrichment score (NES) of $T F A P 2 A$ was 6.802 . e TFAP2A expression was associated with poor survival for patients with CRC. $* p<0.05, * * p<0.01, * * * p<0.001$

ECM [30]. At present, many studies have confirmed that curcumin can regulate EMT pathway, for example, curcumin inhibited cancer cell invasion via downregulating MMPs and uPA [31]; curcumin exhibits antimetastatic properties of melanoma cells by regulating integrin receptors, collagenase activity, and expression of nonmetastatic gene 23 (Nm23), and E-cadherin [32]. However, the role of ECM in colorectal CSC remains unclear and will be investigated in future studies.

After identifying the master regulators that targeted the aforementioned genes, we found that curcumin inhibited LGR5(+) colorectal CSC proliferation by downregulating TFAP2A-mediated ECM-receptor interaction. TFAP $2 A$ is a member of the AP-2 family of transcription factors, and this family is composed of TFAP2A/AP-2 $\alpha$, TFAP2B/ AP- $2 \beta$, TFAP2C/AP- $2 \gamma$, TFAP2D/AP- $2 \delta$, and TFAP2E/ AP-2 $\varepsilon$ [33]. The aberrant expression of TFAP2A has been reported in different cancers [34]. For example, TFAP2A is overexpressed in human nasopharyngeal carcinoma and promotes tumorigenesis by influencing the HIF-1 $\alpha / \mathrm{VEGF} /$ PEDF pathway [35]. However, reduced TFAP2A expression is associated with poor prognosis in gastric cancer [36], and the loss of TFAP2A is linked with melanoma through the regulation of cell adhesion molecules [37]. In addition, Dimitrova found that the expression of TFAP2A is increased in more "stem-like" cancers [38]. Our results have shown that TFAP2A expression was markedly elevated in CRC compared with that in noncancerous colorectal tissues, and was associated with poor prognosis in CRC. Previous studies have found that curcumin induced apoptosis via reducing the expression of AP- $2 \gamma$ in human malignant testicular germ cells, whereas the pretreatment with the proteasome inhibitor MG132 blocked both the curcumin-induced reduction of AP- $2 \gamma$ and antiproliferative effect. Nevertheless, the effect of TFAP2A in CRC has not yet been reported. In future, the role of TFAP2A and other genes in curcumin-induced apoptosis of colorectal CSCs will be studied.

In summary, we found that curcumin suppresses the proliferation of LGR5(+) colorectal CSCs by inducing autophagy and transcriptionally repressing the oncogenic TFAP2A-mediated ECM pathway. Lastly, while our study entailed mostly in vitro experiments, further studies, especially in vivo, are needed to understand the mechanism of effect of curcumin in LGR5(+) colorectal CSCs and to evaluate this potential therapeutic approach to CRC. In addition, the correlation between TFAP2A-mediated ECM pathway and autophagy also deserves further study. 
Table 1 Correlation between TFAP2A expression and clinicopathological characteristics

\begin{tabular}{|c|c|c|c|c|c|c|}
\hline & \multirow[t]{2}{*}{ Variables } & \multicolumn{2}{|c|}{ TFAP2A expression } & \multirow[t]{2}{*}{ Total } & \multirow[t]{2}{*}{$\chi^{2}$} & \multirow[t]{2}{*}{$p$ value } \\
\hline & & Low & High & & & \\
\hline \multirow[t]{3}{*}{ Age (year) } & & & & & 1.786 & 0.181 \\
\hline & $\leq 65$ & 7 & 32 & 39 & & \\
\hline & $>65$ & 2 & 35 & 37 & & \\
\hline \multirow[t]{3}{*}{ Gender } & & & & & 0.001 & 0.970 \\
\hline & Female & 3 & 27 & 30 & & \\
\hline & male & 6 & 40 & 46 & & \\
\hline \multirow[t]{3}{*}{ Grade } & & & & & 0.032 & 0.857 \\
\hline & $\mathrm{I} / \mathrm{II}$ & 6 & 50 & 56 & & \\
\hline & III & 3 & 16 & 19 & & \\
\hline \multirow[t]{3}{*}{ T stage } & & & & & 0.012 & 0.912 \\
\hline & $\mathrm{T} 2 / \mathrm{T} 3$ & 3 & 18 & 21 & & \\
\hline & $\mathrm{T} 4$ & 5 & 45 & 50 & & \\
\hline \multirow[t]{3}{*}{$\mathrm{N}$ stage } & & & & & 0.000 & 1.000 \\
\hline & No & 5 & 36 & 41 & & \\
\hline & N1/N2 & 3 & 28 & 31 & & \\
\hline \multirow[t]{3}{*}{ M stage } & & & & & & 1.000 \\
\hline & M0 & 9 & 66 & 75 & & \\
\hline & M1 & 0 & 1 & 1 & & \\
\hline \multirow[t]{3}{*}{ TNM stage } & & & & & 0.000 & 1.000 \\
\hline & $\mathrm{I} / \mathrm{II}$ & 5 & 36 & 41 & & \\
\hline & III/IV & 3 & 28 & 31 & & \\
\hline
\end{tabular}

Funding This study was supported by Natural Science Foundation of Zhejiang Province (Grant Nos. LY19H280007 and LQ17H310002) and Zhejiang Medical Technology Plan Project (Grant Nos. 2017ZD003 and 2019KY027).

\section{Compliance with ethical standards}

Conflicts of interest The authors declare no conflict of interest.

Open Access This article is licensed under a Creative Commons Attribution 4.0 International License, which permits use, sharing, adaptation, distribution and reproduction in any medium or format, as long as you give appropriate credit to the original author(s) and the source, provide a link to the Creative Commons licence, and indicate if changes were made. The images or other third party material in this article are included in the article's Creative Commons licence, unless indicated otherwise in a credit line to the material. If material is not included in the article's Creative Commons licence and your intended use is not permitted by statutory regulation or exceeds the permitted use, you will need to obtain permission directly from the copyright holder. To view a copy of this licence, visit http://creativecommons.org/licenses/by/4.0/.

\section{References}

1. Dekker E, Tanis PJ, Vleugels JLA, Kasi PM, Wallace MB (2019) Colorectal cancer. The Lancet 394:1467-1480

2. Parizadeh SM, Jafarzadeh-Esfehani R, Hassanian SM, Parizadeh SMR, Vojdani S, Ghandehari M, Ghazaghi A, Khazaei M, Shahidsales S, Rezayi M et al (2019) Targeting cancer stem cells as therapeutic approach in the treatment of colorectal cancer. Int J Biochem Cell Biol 110:75-83

3. Tauriello DV, Calon A, Lonardo E, Batlle E (2017) Determinants of metastatic competency in colorectal cancer. Mol Oncol 11:97-119

4. Zeuner A, Todaro M, Stassi G, De Maria R (2014) Colorectal cancer stem cells: from the crypt to the clinic. Cell Stem Cell 15:692-705

5. Hu YB, Yan C, Mu L, Mi YL, Zhao H, Hu H, Li XL, Tao DD, Wu YQ, Gong JP, Qin JC (2019) Exosomal Wnt-induced dedifferentiation of colorectal cancer cells contributes to chemotherapy resistance. Oncogene 38:1951-1965

6. Tsuda T (2018) Curcumin as a functional food-derived factor: degradation products, metabolites, bioactivity, and future perspectives. Food Funct 9:705-714

7. Nagaraju GP, Benton L, Bethi SR, Shoji M, El-Rayes BF (2019) Curcumin analogs: their roles in pancreatic cancer growth and metastasis. Int J Cancer 145:10-19

8. Giordano A, Tommonaro G (2019) Curcumin and cancer. Nutrients 11(10):2376

9. Hosoya T, Nakata A, Yamasaki F, Abas F, Shaari K, Lajis NH, Morita H (2012) Curcumin-like diarylpentanoid analogues as melanogenesis inhibitors. J Nat Med 66:166-176

10. Yang C, Ma X, Wang Z, Zeng X, Hu Z, Ye Z, Shen G (2017) Curcumin induces apoptosis and protective autophagy in castrationresistant prostate cancer cells through iron chelation. Drug Des Devel Ther 11:431-439

11. Chen P, Huang HP, Wang Y, Jin J, Long WG, Chen K, Zhao $\mathrm{XH}$, Chen CG, Li J (2019) Curcumin overcome primary gefitinib resistance in non-small-cell lung cancer cells through inducing autophagy-related cell death. J Exp Clin Cancer Res 38:254

12. Lee YH, Song NY, Suh J, Kim DH, Kim W, Ann J, Lee J, Baek JH, Na HK, Surh YJ (2018) Curcumin suppresses oncogenicity 
of human colon cancer cells by covalently modifying the cysteine 67 residue of SIRT1. Cancer Lett 431:219-229

13. de Sousa e Melo F, Kurtova AV, Harnoss JM, Kljavin N, Hoeck JD, Hung J, Anderson JE, Storm EE, Modrusan Z, Koeppen H, et al (2017) A distinct role for Lgr5(+) stem cells in primary and metastatic colon cancer. Nature 543:676-680

14. Shimokawa M, Ohta Y, Nishikori S, Matano M, Takano A, Fujii M, Date S, Sugimoto S, Kanai T, Sato T (2017) Visualization and targeting of LGR5(+) human colon cancer stem cells. Nature 545:187-192

15. Zhong Q, Liu ZH, Lin ZR, Hu ZD, Yuan L, Liu YM, Zhou AJ, Xu LH, Hu LJ, Wang ZF et al (2018) The RARS-MAD1L1 fusion gene induces cancer stem cell-like properties and therapeutic resistance in nasopharyngeal carcinoma. Clin Cancer Res 24:659-673

16. An X, Chen L (2018) Flow cytometry (FCM) analysis and fluorescence-activated cell sorting (FACS) of erythroid cells. Methods Mol Biol 1698:153-174

17. Hlosrichok A, Sumkhemthong S, Sritularak B, Chanvorachote P, Chaotham C (2018) A bibenzyl from Dendrobium ellipsophyllum induces apoptosis in human lung cancer cells. J Nat Med 72:615-625

18. Liu LQ, Wang SB, Shao YF, Shi JN, Wang W, Chen WY, Ye ZQ, Jiang JY, Fang QX, Zhang GB, Xuan ZX (2019) Hydroxychloroquine potentiates the anti-cancer effect of bevacizumab on glioblastoma via the inhibition of autophagy. Biomed Pharmacother 118:109339

19. Eskelinen EL, Reggiori F, Baba M, Kovacs AL, Seglen PO (2011) Seeing is believing: the impact of electron microscopy on autophagy research. Autophagy 7:935-956

20. Wang W, Liu L, Zhou Y, Ye Q, Yang X, Jiang J, Ye Z, Gao F, Tan $X$, Zhang $G$ et al (2019) Hydroxychloroquine enhances the antitumor effects of BC001 in gastric cancer. Int J Oncol 55:405-414

21. Poluri RTK, Beauparlant CJ, Droit A, Audet-Walsh E (2019) RNA sequencing data of human prostate cancer cells treated with androgens. Data Brief 25:104372

22. Qi W, Schlapbach R, Rehrauer H (2017) RNA-Seq data analysis: from raw data quality control to differential expression analysis. Methods Mol Biol 1669:295-307

23. Gao Y, Zhang S, Zhang Y, Qian J (2020) Identification of MicroRNA-target gene-transcription factor regulatory networks in colorectal adenoma using microarray expression data. Front Genet 11:463

24. Janky R, Verfaillie A, Imrichova H, Van de Sande B, Standaert L, Christiaens V, Hulselmans G, Herten K, Naval Sanchez M, Potier $\mathrm{D}$ et al (2014) iRegulon: from a gene list to a gene regulatory network using large motif and track collections. PLoS Comput Biol 10:e1003731

25. Zhang F, Gu X, Yi S, Xu H (2019) Dysregulated transcription factor TFAP2A after peripheral nerve injury modulated schwann cell phenotype. Neurochem Res 44:2776-2785

26. Wang D, Fu L, Sun H, Guo L, DuBois RN (2015) Prostaglandin E2 promotes colorectal cancer stem cell expansion and metastasis in mice. Gastroenterology 149(1884-1895):e1884
27. Chiba T, Kamiya A, Yokosuka O, Iwama A (2009) Cancer stem cells in hepatocellular carcinoma: recent progress and perspective. Cancer Lett 286:145-153

28. Wahab SMR, Islam F, Gopalan V, Lam AK (2017) The identifications and clinical implications of cancer stem cells in colorectal cancer. Clin Colorectal Cancer 16:93-102

29. Fu H, Wang C, Yang D, Wei Z, Xu J, Hu Z, Zhang Y, Wang W, Yan R, Cai Q (2018) Curcumin regulates proliferation, autophagy, and apoptosis in gastric cancer cells by affecting PI3K and P53 signaling. J Cell Physiol 233:4634-4642

30. Ragelle H, Naba A, Larson BL, Zhou F, Prijic M, Whittaker CA, Del Rosario A, Langer R, Hynes RO, Anderson DG (2017) Comprehensive proteomic characterization of stem cell-derived extracellular matrices. Biomaterials 128:147-159

31. Yodkeeree S, Chaiwangyen W, Garbisa S, Limtrakul P (2009) Curcumin, demethoxycurcumin and bisdemethoxycurcumin differentially inhibit cancer cell invasion through the down-regulation of MMPs and uPA. J Nutr Biochem 20:87-95

32. Ray S, Chattopadhyay N, Mitra A, Siddiqi M, Chatterjee A (2003) Curcumin exhibits antimetastatic properties by modulating integrin receptors, collagenase activity, and expression of $\mathrm{Nm} 23$ and E-cadherin. J Environ Pathol Toxicol Oncol 22:49-58

33. Chambers BE, Gerlach GF, Clark EG, Chen KH, Levesque AE, Leshchiner I, Goessling W, Wingert RA (2019) Tfap2a is a novel gatekeeper of nephron differentiation during kidney development. Development 146(13):dev172387

34. Zhang N, Li Y, Xie M, Song Y, Liu J, Lei T, Shen Y, Yu J, Yang M (2020) DACT2 modulated by TFAP2A-mediated allelic transcription promotes EGFR-TKIs efficiency in advanced lung adenocarcinoma. Biochem Pharmacol 172:113772

35. Shi D, Xie F, Zhang Y, Tian Y, Chen W, Fu L, Wang J, Guo W, Kang T, Huang W, Deng W (2014) TFAP2A regulates nasopharyngeal carcinoma growth and survival by targeting HIF- $1 \alpha$ signaling pathway. Cancer Prev Res (Phila) 7:266-277

36. Cao L, Wang S, Zhang Y, Wong KC, Nakatsu G, Wang X, Wong S, Ji J, Yu J (2018) Zinc-finger protein 471 suppresses gastric cancer through transcriptionally repressing downstream oncogenic PLS3 and TFAP2A. Oncogene 37:3601-3616

37. Hallberg AR, Vorrink SU, Hudachek DR, Cramer-Morales K, Milhem MM, Cornell RA, Domann FE (2014) Aberrant CpG methylation of the TFAP2A gene constitutes a mechanism for loss of TFAP2A expression in human metastatic melanoma. Epigenetics 9:1641-1647

38. Dimitrova Y, Gruber AJ, Mittal N, Ghosh S, Dimitriades B, Mathow D, Grandy WA, Christofori G, Zavolan M (2017) TFAP2A is a component of the ZEB1/2 network that regulates TGFB1-induced epithelial to mesenchymal transition. Biol Direct $12: 8$

Publisher's Note Springer Nature remains neutral with regard to jurisdictional claims in published maps and institutional affiliations.

\section{Authors and Affiliations}

\section{Xiaohong Mao ${ }^{1} \cdot$ Xin Zhang $^{2} \cdot$ Xiaowei Zheng $^{3} \cdot$ Yongwu Chen $^{4} \cdot$ Zixue Xuan $^{1} \cdot$ Ping Huang ${ }^{1}$}

Zixue Xuan

xuanzixue0222@163.com

Ping Huang

huangping_zry@163.com
1 Department of Pharmacy, Zhejiang Provincial People's Hospital, People's Hospital of Hangzhou Medical College, Hangzhou 310014, China 
2 Department of Pathology, Zhejiang Provincial People's Hospital, People's Hospital of Hangzhou Medical College, Hangzhou 310014, China

3 Department of Pharmacy, Zhejiang Cancer Hospital, Hangzhou 310022, China
4 Department of Pharmacy, Division of Life Sciences and Medicine, The First Affiliated Hospital of USTC, University of Science and Technology of China, Hefei 230036, China 